\title{
«Une MRC - une ville» à Rouyn-Noranda: de nouveaux rapports (de force) entre l'urbain et le rural ?
}

\author{
Guy Chiasson ${ }^{1}$ \\ Université du Québec à Hull
}

Ce texte présente et analyse le modèle " une MRC - une ville » qui préside à la réorganisation municipale de la MRC de Rouyn-Noranda. Il veut montrer que la mise en application de ce modèle risque de modifier les rapports de force entre la ville centre et sa périphérie rurale de façon à fortement affaiblir le pouvoir rural.

L'agglomération de Rouyn-Noranda fait partie des 25 agglomérations de recensement ciblées par la Loi 124 devant ainsi être fusionnées ${ }^{2}$. Les élus locaux de la MRC, réunis dans un colloque au mois de novembre 2000, ont rejeté de façon unanime le scénario d'une fusion à l'échelle de l'agglomération en faisant valoir que celui-ci risque de concourir à la marginalisation des petites municipalités rurales hors-agglomération. Ils ont donc demandé un délai à la ministre Louise Harel afin d'étudier deux scénarios alternatifs de fusion: un premier qui favoriserait la création de cinq municipalités à même le territoire de la MRC et un second qui est celui d'une MRC - une ville. Après d'importantes négociations, c'est ce dernier scénario qui a été retenu autant par une majorité d'élus locaux (10 des 13 maires de la MRC ont accepté le scénario une $M R C$ - une ville) que par le rapport du mandataire remis à la ministre en avril 2001 et finalement par le gouvernement ${ }^{3}$.

En instaurant une nouvelle structure à un seul palier, le projet une MRC - une ville de Rouyn-Noranda ne manquera pas de transformer les rapports de pouvoir entre la ville centre et sa périphérie largement rurale. Quelle sera la place de l'urbain et des espaces ruraux dans cette nouvelle ville-MRC?

Plus spécifiquement, quels impacts aura l'abolition des MRC sur les pouvoirs respectifs de la ville centre et de la périphérie rurale?

Ce modèle retiendra notre attention parce qu'en plus de redessiner profondément la carte municipale il rompt avec la gouverne locale à deux paliers (municipalités locales et MRC) instituée il y a de cela plus de deux décennies par la Loi $125^{4}$. En instaurant une nouvelle structure à un seul palier, le projet une MRC - une ville de RouynNoranda ne manquera pas de transformer les rapports de pouvoir entre la ville centre et sa périphérie largement rurale $^{5}$. Quelle sera la place de l'urbain et des espaces ruraux dans cette nouvelle ville-MRC ? Plus spécifiquement, quels impacts aura l'abolition des MRC sur les pouvoirs respectifs de la ville centre et de la périphérie rurale? Nous aborderons ces questions en analysant plus spécifiquement le rapport du mandataire Jean-Charles Coutu (2001) ${ }^{6}$. Ce rapport, puisqu'il a esquissé les formes que pourrait prendre la nouvelle structure municipale, servira de vitrine pour analyser la signification pour le couple rural-urbain du modèle une MRC - une ville.

Le modèle une MRC - une ville met en place des modalités de gouverne qui risquent fort d'accélérer la marginalité du pouvoir rural dans les structures décisionnelles locales en permettant à la ville centre de peser de tout son poids démographique.

Après avoir présenté les grandes lignes du modèle une $M R C$ - une ville, nous nous arrêterons sur les conséquences de ce modèle sur la dynamique rurale-urbaine. Plus précisément, nous argumentons que le modèle une $M R C$ une ville met en place des modalités de gouverne qui risquent fort d'accélérer la marginalité du pouvoir rural dans les structures décisionnelles locales en permettant à la ville centre de peser de tout son poids démographique. 


\section{Le modèle une $M R C$ - une ville: de nouvelles structures pour dépasser le conflit urbain-rural ?}

Le modèle une MRC - une ville représente un scénario assez innovateur autant par rapport à ce qui s'est fait ailleurs que par rapport à ce qui était prévu dans la Loi 124 (la fusion obligatoire à l'échelle des agglomérations de recensement (AR)). Suivant ce modèle, l'ensemble des treize municipalités de la MRC devront être regroupées dans la nouvelle ville de Rouyn-Noranda. C'est donc un territoire beaucoup plus étendu que celui de l'AR qui sera fusionné afin d'inclure six municipalités hors-agglomération. Dans l'esprit du mandataire, cette fusion signifie non seulement la création d'une seule municipalité pour l'ensemble du territoire MRC mais doit nécessairement aboutir à l'abolition de la MRC comme palier de gouvernement et au transfert de ses responsabilités vers la nouvelle ville.

Dans l'optique d'un regroupement de toutes les municipalités du territoire actuellement couvert par la MRC de Rouyn-Noranda, il me semble évident que l'existence même de la MRC comme structure administrative n' aura plus sa raison d'être. C'est la nouvelle ville qui devra assumer toutes les responsabilités actuelles de la MRC (p. 21).

Déjà, on peut constater que le modèle une $M R C$ - une ville apporte une double mutation de la gouverne locale: l'intégration de treize municipalités dans une mais aussi le rapatriement des compétences de la MRC au palier municipal.

À ces bouleversements à l'échelle du territoire de la MRC se rajoute la création de structures inédites à l'échelle des anciennes localités. Afin d'assurer une certaine vie communautaire dans ces localités, le mandataire préconise la mise sur pied de «secteurs locaux » à la place des anciennes municipalités ou des anciens quartiers de la ville de Rouyn-Noranda ${ }^{7}$. Pour chacun de ces secteurs est proposée la création d'un organisme paramunicipal : la Corporation de services communautaires et qualité de vie. Cet organisme, redevable au Conseil municipal mais élu par la population du secteur, serait chargé d'animer la vie communautaire, de soutenir certains projets locaux et d'assurer le maintien de certains services de proximité (p. 37).

De façon intéressante, le mandataire justifie en bonne partie le choix d'une fusion à l'échelle de la MRC par la nécessité de mieux arrimer les milieux urbain et rural de la MRC. «J'estime que cet équilibre partenarial entre le milieu urbain et le milieu rural doit être recherché et atteint a fortiori dans le cas du regroupement des municipalités urbaines et rurales. » (p. 19). Le rapport suggère ainsi que la présence de municipalités urbaines et de municipalités rurales est une source de rivalités et de «méfiance» qui nuisent au bien commun de l'ensemble du territoire $\mathrm{MRC}^{8}$. La fusion de l'ensemble de la MRC, contrairement à d'autres scénarios, permettrait de résorber ces rivalités :

Parmi les scénarios de regroupements, on aurait pu préconiser un regroupement de toutes les municipalités rurales qui ceinturent le milieu urbain de Rouyn-Noranda. Cela n'aurait probablement que perpétué une rivalité malsaine entre le milieu rural et le milieu urbain et augmenté le clivage entre les citoyens urbains et ruraux et ce, au détriment du développement économique et social de toute la MRC (p. 19).

Dans un même ordre d'idée, la mise au rancart de la MRC et le transfert de ses responsabilités vers la ville sont aussi rendus nécessaires du fait du clivage urbainrural. La MRC est caractérisée d' «institution dépassée » par le mandataire du fait des conflits qu'elle génère entre les milieux ruraux et urbains (p. 21).

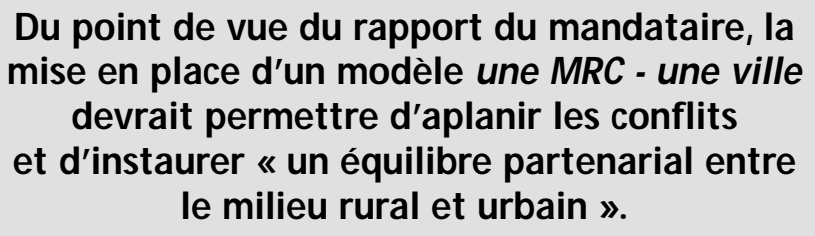

Du point de vue du rapport du mandataire, la mise en place d'un modèle une $M R C$ - une ville devrait permettre d'aplanir les conflits et d'instaurer « un équilibre partenarial entre le milieu rural et urbain ». Cependant, comme le suggère Jean-Pierre Collin, les milieux ruraux et urbains renvoient à des réalités très différentes et leur regroupement ne se fait pas sans problème: «comment faire cohabiter les perspectives urbaines et rurales quand les deux univers diffèrent tellement, autant par la taille démographique que par leur organisation territoriale ? $»^{9}$. À l'instar de Collin, il nous semble que l'intégration du rural et de l'urbain dans une même structure politique ne se fera pas sans heurts et surtout qu'elle risque d'avoir des conséquences imprévues par le rapport Coutu. Nous 
voulons montrer qu'il y a lieu de craindre que la mise en application de ce modèle, plutôt que de favoriser un partenariat urbain-rural, va renforcer l'emprise urbaine sur les institutions locales au détriment du pouvoir des ruraux.

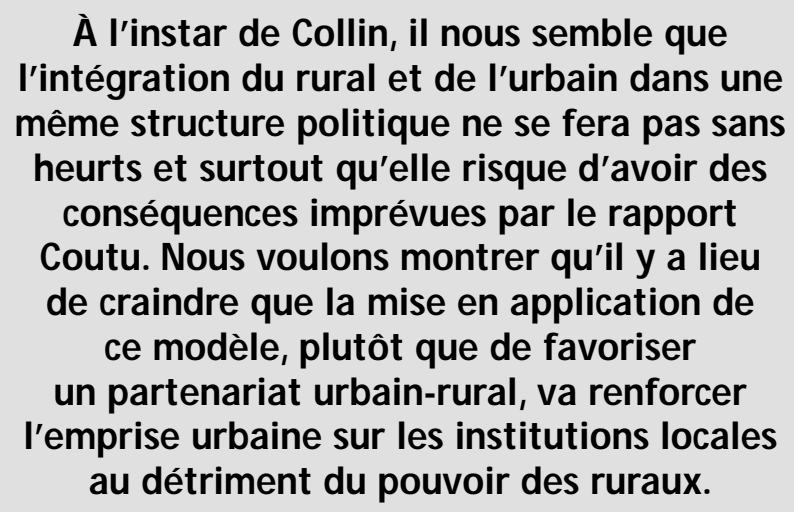

\section{D es différences entre le rural et l'urbain}

La différence démographique identifiée par Collin est particulièrement évidente dans le cas de la MRC de Rouyn-Noranda. Un regard rapide sur les statistiques de populations permet de voir des écarts de population impressionnants entre la ville centre et sa périphérie. Comme on peut le constater à la lecture du tableau 1, la plupart des municipalités périphériques affichent des populations très faibles (moins de 1000 habitants) - sauf McWatters, Évain et dans une moindre mesure D' Alembert ${ }^{10}$ - comparativement à la ville centre qui elle compte près de 40000 habitants. L'ampleur du déséquilibre démographique apparaît de façon encore plus crue lorsque l'on tient compte du fait que la ville centre englobe plus de $70 \%$ de la population de la MRC tandis que les six municipalités rurales hors-agglomérations regroupées représentent $8,5 \%$ de cette population.

\section{Rétablir le pouvoir politique urbain}

Ces déséquilibres démographiques rendent, dans le meilleur des cas, la tâche difficile à ceux qui veulent accorder un poids politique substantiel aux milieux ruraux face aux villes centres. Cependant, dans une structure à deux paliers, les déséquilibres démographiques identifiés plus haut se retrouvaient quelque peu atténués, notamment par la présence des MRC. La création des MRC visait initialement à mettre dos à dos les villes régionales et leurs périphéries rurales et ainsi instaurer un certain équilibre entre elles ${ }^{11}$. Avec le temps les MRC, incluant celle de Rouyn-Noranda, sont devenues des lieux où les milieux ruraux peuvent exercer un pouvoir significatif ${ }^{12}$.

Tableau 1. Population des municipalités de la MRC de Rouyn-Noranda

\begin{tabular}{llcc}
\hline & Municipalités & Population 2001 & \% de la population de la MRC \\
\hline Agglomération de & Arntfield & 436 & 1 \\
recensement (AR) & Bellecombe & 786 & 1,8 \\
& Cloutier & 370 & 0,8 \\
& D'Alembert & 958 & 2,2 \\
& Évain & 3919 & 9,1 \\
& McWatters & 2058 & 4,8 \\
& Rouyn-Noranda & 3099 & 71,7 \\
& Ensemble de l'AR & 39517 & 91,4 \\
\hline \multirow{2}{*}{ Autres municipalités } & Cadillac & 952 & 2,2 \\
de la MRC & Cléricy & 502 & 1,2 \\
& Destor & 488 & 1,1 \\
& Montbeillard & 801 & 1,8 \\
& Mont-Brun & 552 & 1,3 \\
& Rollet & 406 & 0,9 \\
& Territoires non-organisés & 7 & 0.02 \\
& Ensemble de la MRC & 43218 & 100 \\
\hline
\end{tabular}


Le principe de la double majorité ${ }^{13}$ qui gouverne le palier MRC, s'il est porteur de conflits et de blocages entre la ville et les municipalités rurales, a aussi l'avantage de forcer les villes-centres à tenir compte des visées des communautés rurales dans des dossiers qui sont considérés comme ayant une portée supralocale ou autrement dit sur des questions qui sont d'intérêt commun à la ville centre et à sa périphérie ${ }^{14}$. La structure MRC accorde ainsi aux milieux ruraux une capacité effective de participer aux décisions de portée supralocale et cela, malgré la faiblesse de leur poids démographique.

De toute évidence, le modèle une MRC - une ville change la donne puisqu'il se rabat sur un seul palier de gouvernement pour l'ensemble de la MRC. Ces changements sont lourds de signification pour la gouverne locale. Si le modèle de gouverne locale à deux paliers permettait d'endiguer le déclin du pouvoir rural, sa remise en question permettra sans doute à la ville centre de peser de tout son poids sur la structure municipale unique. Pour s'en convaincre, il s'agit de regarder le découpage électoral proposé par le rapport Coutu pour la nouvelle ville.

\section{La structure MRC accorde ainsi aux milieux ruraux une capacité effective de participer aux décisions de portée supralocale et cela, malgré la faiblesse de leur poids démographique.}

Le mandataire constate que la Loi sur les élections et les référendums dans les municipalités fixe à $15 \%$ l'écart maximum de population entre deux districts électoraux d'une même ville. Le respect d'une telle règle rend difficile l'accord d'un siège électoral à chacune des anciennes municipalités : certains districts urbains existants comptent plus de 2600 électeurs tandis que la plupart des municipalités rurales de la MRC en comptent moins de 700. Il proposa donc la solution suivante: jusqu'en 2002 chacune des anciennes municipalités tout comme les anciens districts de la ville centre recevront un district électoral. Après les élections de 2002, le nombre de districts devrait être resserré afin de mieux respecter le poids démographique des communautés, ce qui impliquera une diminution importante du nombre de sièges ruraux sur le conseil de la nouvelle ville. À terme, 8 des 14 districts électoraux seraient issus du territoire de l'ancienne ville centre (p. 31). Inversement, dans la portion rurale de la MRC, 10 anciennes municipalités seraient regroupées à l'intérieur de trois districts électoraux ${ }^{15}$. Le découpage électoral proposé démontre bien une volonté d'amener une représen- tation politique qui soit plus conséquente avec le poids démographique respectif des ruraux et des urbains. Les conséquences très pratiques en sont qu'une majorité des voix sur le conseil municipal reviendra à la population urbaine et que les anciennes communautés rurales auront relativement peu d'emprise sur ce conseil. Il y a fort à craindre qu'étant donné la faiblesse démographique du rural, le «partenariat urbain-rural» sera marqué par des rapports de force nettement à l'avantage de l'urbanité.

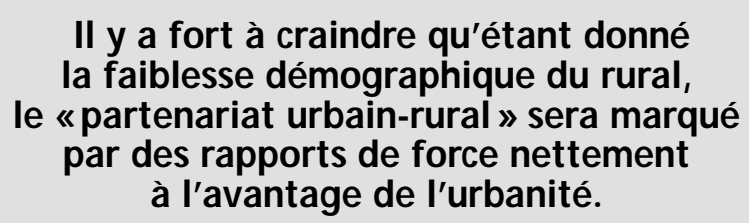

\section{Une perte d'emprise sur les enjeux supralocaux}

Il faut tout de même tenir compte du fait que le rapport du mandataire reconnaît les anciennes communautés en leur accordant le statut de secteur. Tel qu'expliqué plus haut, selon le modèle une $M R C$ - une ville, chaque secteur sera appelé à élire un conseil responsable de la vie communautaire (bénévolat, associations locales) et de services de proximité dans la communauté (p. 31-32). Cette formule est intéressante dans la mesure où elle instaure un pouvoir des communautés sur des enjeux locaux qui leur sont propres. De l'avis du mandataire, il s'agit également d'une façon de tenir compte du sentiment d'appartenance locale, de la participation de la communauté et de permettre le maintien de services (souvent bénévoles) déjà présents dans la communauté (p. 30). Cette formule est intéressante non seulement parce qu'elle préserve un certain pouvoir pour les communautés rurales mais également parce qu'elle instaure des mécanismes faisant en sorte que les quartiers urbains soient aussi amenés à gérer leurs services de proximité à l'échelle locale.

Au-delà de l'intérêt de cette formule de secteurs, on doit tout de même convenir qu'elle fait partie d'un recentrage du pouvoir rural vers des enjeux de portée beaucoup plus réduite. Dans le contexte d'une gouverne locale à deux paliers, les ruraux pouvaient prétendre exercer un certain contrôle à travers le palier MRC sur des enjeux d'intérêt commun pour la ville et la campagne. En remettant à une ville unique les responsabilités de la MRC, ces décisions relèvent de plus en plus d'une entité municipale que les ruraux contrôleront peu. La mise en place des secteurs 
institue un lieu où les ruraux ont un certain pouvoir mais ce pouvoir porte uniquement sur des enjeux de nature localisée et ne permet donc pas de pallier à cette situation.

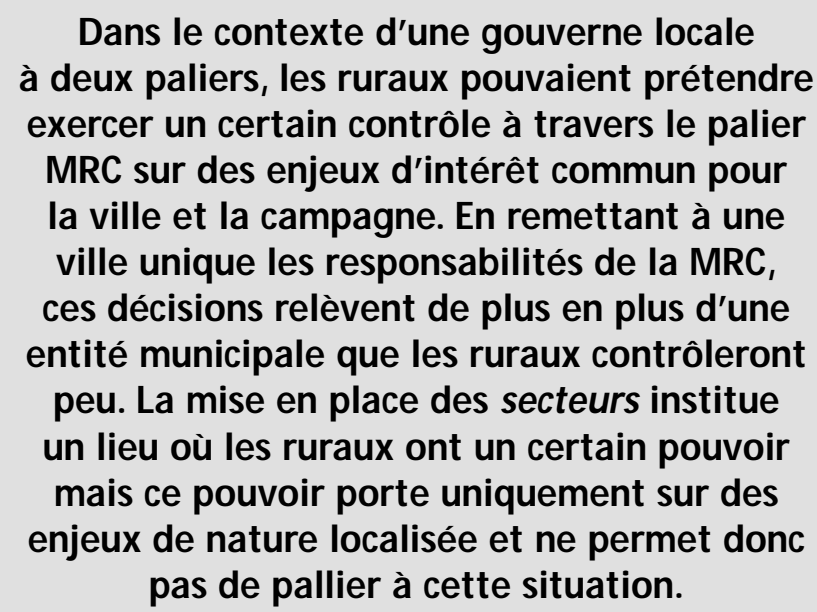

\section{Conclusion}

Ce petit texte avait comme double objectif de présenter le modèle particulier de fusion municipale préconisé pour la MRC de Rouyn-Noranda et d'en évaluer les conséquences sur la dynamique rurale-urbaine. Ce modèle ira plus loin que ce qui est exigé par la Loi 124 et proposera à la fois une fusion de l'ensemble des municipalités de la MRC et l'abolition de la MRC. De l'avis du mandataire, ces mesures devraient permettre d'instaurer «un équilibre partenarial entre les milieux urbains et ruraux ». Nous avons voulu montrer que cette lecture de l'impact de la fusion à Rouyn-Noranda est fortement optimiste. À notre avis, la mise en place du modèle une MRC - une ville, loin de permettre un partenariat urbain-rural équilibré, va plutôt favoriser un rapport de force nettement à l'avantage de la ville.

Ce modèle instaure des structures municipales qui sont plus portées que ne l'étaient les MRC à refléter le poids démographique dominant de la ville et la faiblesse du rural à ce chapitre. D'une part, le découpage électoral proposé par le mandataire laisse bien transparaître une volonté de rétablir le poids politique des urbains en fonction de leur poids démographique, ce qui laisse présager que le pouvoir des ruraux sur le conseil municipal soit lui aussi faible. D'autre part, ce modèle contribue à recentrer le pouvoir rural vers des enjeux plus localisés. La portion de pouvoir sur les enjeux supralocaux que les communautés rurales exerçaient auparavant par l'entre- mise de la MRC va en bonne partie leur échapper puisqu'ils relèveront du conseil municipal.

\section{Notes et références}

1 Guy Chiasson est professeur au Département de travail social à l'Université du Québec à Hull.

2 MINISTÈRE DES AFFAIRES MUNICIPALES ET DE LA MÉTROPOLE (MAMM) (2000). La réorganisation municipale: Changer les façons de faire pour mieux servir les citoyens, Québec.

3 COUTU, J.C. (2001). Rapport sur la réorganisation municipale dans la Municipalité régionale de comté de Rouyn-Noranda, avril, (rapport du mandataire à la ministre Louise Harel disponible sur le site du Ministère des Affaires municipales et de Métropole), p. 7-8.

4 DÉSY, J. (1988). Municipalités et MRC: vers une union libre?, Chicoutimi, Groupe de recherche et d'intervention régionales.

5 Pour une présentation des conséquences de la réforme municipale sur les rapports entre l'urbanité et la ruralité, voir le texte de Jean-Pierre COLLIN (2001). «Le milieu rural et les MRC en attente de leur réforme», dans Roch Côté (dir.), Québec 2002. Annuaire politique, social, économique et culturel, Montréal, Fides, p. 235-242.

6 Comme ce fut le cas pour les autres agglomérations de recensement, la ministre Louise Harel nomma un mandataire (JeanCharles Coutu) chargé après un travail de consultation avec le milieu de proposer un scénario de fusion. Son rapport fut soumis à la ministre au mois d'avril 2001: COUTU, J.C. (2001). Op. cit., note 3 . Dorénavant lorsque nous nous référerons à ce rapport, nous n'indiquerons que la page de référence entre parenthèses.

7 Le mandataire va préférer la notion de «secteur» à celle d' «arrondissement » mise de l'avant dans le contexte montréalais puisque cette dernière notion supposerait une allocation de pouvoirs plus large que celle de secteur (p. 25).

8 Il est intéressant de constater que le mandataire Coutu reprend ici en bonne partie l'argumentaire du gouvernement en le modifiant quelque peu. On se souviendra que selon le Livre blanc, la fragmentation municipale à l'intérieur des agglomérations municipales est le problème central que les fusions veulent régler (MAMM, 2000). Tout comme le Livre blanc, le mandataire Coutu a mis en évidence les méfaits de la fragmentation; dans son cas, c'est cependant la fragmentation à l'échelle de la MRC plutôt qu'à celle de l'agglomération urbaine qui est montrée du doigt. Jean-Pierre Collin (2001) fait d'ailleurs remarquer que le rapport Coutu n'est pas le seul à s'aligner sur la position gouvernementale. La plupart des rapports des mandataires ont calqué les grandes lignes de l'argumentaire gouvernemental.

9 COLLIN, J.P. (2001). Op. cit., note 5, à la page 236.

10 Ces trois municipalités ceinturent la ville de Rouyn-Noranda et peuvent être qualifiées de périurbaine avec des populations un peu plus importantes et des taux de croissance de population 
nettement plus élevés. Le mandataire va d'ailleurs les qualifier de faisant partie du « secteur urbain ».

11 DÉSY, J. (1988). Op. cit., note 4.

12 CARRIER, M. et B. JEAN (2000). « La reconstruction de la légitimité des collectivités rurales », dans Mario Carrier et Serge Côté (dir.), Gouvernance et territoires ruraux. Éléments d'un débat sur la responsabilité du développement, Québec, Presses de l'Université du Québec, p. 41-63.

13 Pour qu'une décision du conseil de MRC soit effective, il faut qu'elle obtienne la majorité des votes des municipalités représentant la majorité de la population de la MRC.

14 Comme le font remarquer plusieurs auteurs, les MRC ont largement dépassé leur responsabilité initiale d'aménage- ment du territoire pour prendre en charge plusieurs services ou dossiers considérés d'intérêt supralocal : gestion des déchets, recyclage, développement économique, tourisme etc. (Carrier et Jean (2000), Op. cit., note 12, p. 42 ; PROULX, M.U. (1992). «Appartenance, utilités, fonctions. Les milieux MRC du Québec », Revue canadienne des sciences régionales, vol. XV, $\mathrm{n}^{\circ}$ 2, septembre, p. 307-325 ; SOKOLOFF, B. (1989). «La place des MRC dans les dynamismes régionaux», Revue canadienne des sciences régionales, vol. XII, $\mathrm{n}^{\circ} 1$, printemps, p. 45-60.

15 Un premier district regrouperait Arntfield, Montbeillard et Rollet, un second inclurait Bellecombe, Beaudry et Cloutier tandis que le troisième serait constitué de D'Alembert, Destor, Cléricy et Mont-Brun. Voir le tableau 5 dans le rapport Coutu. 